\title{
Concavity and Monotonicity Properties in a Groundwater Management Model
}

\author{
Woonghee Tim Huh* \\ Chandra Kiran Krishnamurthy ${ }^{\dagger}$ \\ Richard Weber
}

\begin{abstract}
We consider a discrete-time groundwater model in which the cost of pumping takes a slightly different form to that which has been traditional in the research literature to date. This enables us to prove that (a) the optimal pumping quantity is nondecreasing in the ground water stock, (b) the stock level remaining after each period's pumping is also nondecreasing in the groundwater stock, (c) the optimal decision is determined by maximizing a concave function, and finally (d) the optimal pumping quantity is nonincreasing in the number of periods to go. We show that (a)-(c), while intuitive, do not hold under traditional modeling assumptions. We also explain the connections between our results and similar ones for some classic problems of operations research.
\end{abstract}

\footnotetext{
*Sauder School of Business, University of British Columbia, Vancouver, BC, Canada, V6T 1Z4. Research supported partially by the National Science Foundation of the United States (DMS-0732169), and the Natural Sciences and Engineering Research Council of Canada, as well as a Pepsico Foundation funding for the project entitled Improving Rural Water and Livelihood Outcomes in India, China, Africa and Brazil.

${ }^{\dagger}$ School of International and Public Affairs, Columbia University, 420 West 118th Street New York, NY 10027, USA

${ }^{\ddagger}$ Statistical Laboratory, University of Cambridge, Wilberforce Road, Cambridge CB3 0WB, United Kingdom
} 


\section{Introduction}

In this paper, we consider the problem of managing groundwater under random recharge in a single cell aquifer. There are two main modeling paradigms used in the literature: the first based on optimal control of the continuous-time model, e.g., [1, 5, 11, 12, 15, 16, 18, 19, 22], and the second based on the dynamic programming formulation of the discrete-time model, e.g., $[7,8,9,10,13,14,21]$. In this paper, we focus on the second paradigm. While several dynamic programming formulations have been proposed in the literature, few structural properties for this problem have been established (although they have been conjectured).

Suppose that at the start of a pumping period the groundwater stock is $x$. Let $C(x, w)$ denote the cost of pumping out a quantity of water $w$ during the current period, given that the stock level of water at the beginning of the period is $x$. It has been traditional in the literature to make the simplifying assumption that

$$
C(x, w)=c(x) w
$$

for a suitably defined nonnegative nonincreasing function $c$. Note two features of this model: the cost $C(x, w)$ depends only on the groundwater stock at a particular instant (e.g., the beginning of the period), not accounting for the fact the stock changes throughout the period as water is being pumped, and the marginal (per-unit) cost of pumping $\partial C / \partial w$ is independent of the quantity of water pumped. We establish that, far from simplifying (presumably the initial intention), the modeling assumption (1) makes it more difficult to establish structural properties. In this paper, we use a more realistic cost function:

$$
C(x, w)=\int_{z=x-w}^{x} \gamma(z) d z
$$

where $\gamma$ is assumed to be nonnegative, nonincreasing and convex. This form of the cost function takes account of changes in the groundwater stock that occur during the pumping period, and further, the marginal cost of pumping depends, in general, on the quantity of water pumped. Let us suppose that time proceeds forward in $T$ discrete periods, denoted $t=1,2, \ldots, T$. Let $w_{t}^{*}(x)$ denote the quantity of water which it is optimal to pump in period $t$, when at the start of that period the groundwater stock is $x$. (If the optimal quantity is not unique, we take the supremum of the optimizers.) Despite model (2) appearing more complicated than model (1), this modification of the cost function allows us to show that the following properties hold: 
- Property (a): $w_{t}^{*}(x)$ is a maximizer of a concave function of $w$.

- Property $(\mathrm{b}): w_{t}^{*}(x)$ is nondecreasing in $x$,

- Property (c): $x-w_{t}^{*}(x)$ is nondecreasing in $x$, and

- Property $(\mathrm{d}): w_{t}^{*}(x)$ is nondecreasing in $t$.

We show in Section 3 that all these properties hold for model (2). This is in contrast to model (1), for which only (c) has been shown to hold, and that with the help of an additional condition on c (for example, as in [14]). The underlying assumption of [13] is the supermodularity of $B(x-y)-C(x, x-y)$ in $(x, y)$, where $B(w)$ is the benefit of withdrawing $w$ units of water, but this condition sometimes fails for model (1); however, for (2), this supermodularity condition always holds, thereby enabling Property (c) to hold. We show by example that Properties (a), (b) and (c) may not hold under (1). (We do not have an example showing (d) failing under (1).)

A common way to prove properties such as (a)-(d) is via a dynamic programming analysis which uses the concavity of the value function in an essential way. We use this approach, with the slight variation that it is the concavity of the value function plus an expression involving the current state that is used in the analysis.

\section{Model}

We describe a discrete-time stochastic groundwater management model, based on the classical paper by Burt [7], which is standard in the literature (see the list of papers cited in Section 1). We consider a finite-horizon problem where $T$ is the planning horizon, and periods are indexed forward by $t \in\{1,2, \ldots, T\}$. Let $x_{t}$ denote the groundwater stock at the beginning of period $t$. The manager decides the withdrawal quantity $w_{t} \geq 0$. Then a non-negative random variable corresponding to the recharge to the groundwater stock, $R_{t}$, is realized. We suppose that the $R_{t}$ 's are independent and identically distributed. We assume that the state transition for the groundwater stock level is given by:

$$
x_{t+1}=x_{t}-w_{t}+R_{t} .
$$


The single-period benefit (or reward) is

$$
G(x, w)=B(w)-C(x, w)
$$

where $B(w)$ is the benefit of withdrawing $w$ units of water and $C(x, w)$ is the cost of pumping. We suppose that $B(w)$ is a concave and nondecreasing function of $w$ and that $C(x, w)$ is of one of the forms in (1) or (2).

The objective is to maximize the present value of the benefit

$$
\sum_{t=1}^{T} \delta^{t} G\left(x_{t}, w_{t}\right)
$$

where $\delta \in(0,1]$ is the discount factor. Then, the value function of dynamic programming is given by the following recursion:

$$
V_{t}\left(x_{t}\right)=\max _{w_{t} \geq 0}\left\{G\left(x_{t}, w_{t}\right)+\delta E\left[V_{t+1}\left(x_{t}-w_{t}+R_{t}\right)\right]\right\}
$$

where $V_{T+1}\left(x_{T+1}\right)=0$. We note the physical property of an aquifer is such that there is no explicit "bottom" - that is, it is always possible to drill deeper into the aquifer and pump more water, though it soon becomes prohibitively expensive at some point depending on the geological structure; thus, without loss of generality, the groundwater stock level $x_{t}$ may be treated as a relative measure, and can be negative. ${ }^{1}$

We comment on the pumping cost $C(x, w)$, which depends on both the quantity of water withdrawn and the pumping cost per unit of water. In models used in the literature (for example, [1], [6], [14], [15], [16], [21] and [22]), the cost function has two characteristics:

- the per-unit cost of pumping, $\partial C(x, w) / \partial w$, depends only on the stock of groundwater at a particular point in time, ignoring the fact that the groundwater stock changes (falls) as pumping progresses within a period - for example, it is based on the groundwater stock at the beginning of the period, and

- the per-unit cost of pumping, $\partial C(x, w) / \partial w$, is independent of $w$, the quantity of water withdrawn.

\footnotetext{
${ }^{1}$ Theorem 1 can also be proved under the constraint of a "bottom", i.e., with a constraint that $x_{t}$ must be maintained nonnegative. To see this, note that by adjusting $\gamma(x)$ so that has its required properties, but tends very rapidly to $-\infty$ as $x$ decreases from 0 towards $-\infty$, we can ensure that under an optimal policy the groundwater stock remains nonnegative.
} 
The first characteristic above is reasonable if the length of a period is sufficiently small and decisions are made frequently, in which case continuous-time models are more appropriate. The second characteristic is a modeling assumption and is not always in accord with hydrologic facts. The most popular form of the cost function used in the literature is that of (1), i.e., $C(x, w)=c(x) w$ for some function $c$, and exhibits both characteristics noted above. (To our knowledge, all numerical examples given in the literature follow this form. This assumption is implicit in almost all studies, and is made clear only in [21].) With this cost function, structural properties such as the concavity of the optimization problem in each period have not been established.

ExAmple. Under model (1), suppose that $B(w)=5 w-w^{2} / 4$ for $w \in[0,10]$, and $B(w)=25$ for $w \geq 10$. Let $c(x)=10-x$ and $T=2$. Then it is an easy calculation that $w_{2}^{*}\left(x_{2}\right)=2 \max \left[0, x_{2}-5\right]$. So, for $5 \leq x_{2} \leq 10$, we have $x_{2}-w_{2}^{*}\left(x_{2}\right)=10-x_{2}$. Thus, property (c) does not hold.

Continuing analysis of this example, it follows that $V_{2}\left(x_{2}\right)=\left(x_{2}-5\right)^{2}$ if $5 \leq x_{2} \leq 10$, and $V_{2}\left(x_{2}\right)=0$ if $x_{2} \leq 5$. Suppose that $\delta=1$ and $R_{1}=0$ with probability 1 . Then, for example, $V_{1}(6)=\arg \max _{w_{1}} H\left(w_{1}\right)$, where

$$
H\left(w_{1}\right)= \begin{cases}B\left(w_{1}\right)-c(6) w_{1}+\left(6-w_{1}-5\right)^{2} & \text { if } w_{1} \in[0,1) \\ B\left(w_{1}\right)-c(6) w_{1} & \text { if } w_{1} \in[1,5]\end{cases}
$$

It is easy to check that the function $H\left(w_{1}\right)$ is not concave in the region $w_{1} \in[0,5]$ (in fact, it has two maximizers $\{0,2\}$ ). Thus, property (a) does not hold.

EXAMPLE. We change the above example by modifying

$$
c(x)= \begin{cases}2 & \text { if } x<2 \\ \epsilon & \text { if } x \geq 2\end{cases}
$$

for $\epsilon>0$, and $R_{1}=1$ with probability 1 . Then, the two-period reward we maximize over $w_{1}$ and $w_{2}$ given $x_{1}$ is

$$
B\left(w_{1}\right)-c\left(x_{1}\right) w_{1}+B\left(w_{2}\right)-c\left(x_{1}-w_{1}+1\right) w_{2} .
$$

For $x_{1}=0.5$, we have $c\left(x_{1}\right)=2$ and $c\left(x_{1}-w_{1}+1\right)=2$ regardless of the value of that we choose for $w_{1}$. Thus, the optimal choice for both $w_{1}$ and $w_{2}$ is 6 . For $x_{1}=1$, we can guarantee that $c\left(x_{1}-w_{1}+1\right)=\epsilon$ if we take $w_{1}=0$, and then we can take $w_{2}=10$, achieving the reward of $25-10 \epsilon$. Taking $w_{1}>0$ produces at most a reward that is at most 
$B(6)-2 \cdot 6=9$ in each period since $c\left(x_{1}-w_{1}+1\right)=2$. Thus, for sufficiently small $\epsilon$, $w_{1}^{*}(0.5)=6$ and $w_{1}^{*}(1)=0$, in contradiction to $(\mathrm{b})$.

\section{Analysis}

Recall from (2) our cost function $C(x, w)=\int_{z=x-w}^{x} \gamma(z) d z$. From the definitions of $V_{t}$ and $G$, we can write $V_{t}\left(x_{t}\right)=\max _{w_{t} \geq 0} U_{t}\left(x_{t}, w_{t}\right)$, where

$$
U_{t}\left(x_{t}, w_{t}\right)=B\left(w_{t}\right)-\int_{z=x_{t}-w_{t}}^{x_{t}} \gamma(z) d z+\delta E\left[V_{t+1}\left(x_{t}-w_{t}+R_{t}\right)\right] .
$$

For any $x_{t}$, let $w_{t}^{*}\left(x_{t}\right)=\arg \max _{w_{t} \geq 0} U_{t}\left(x_{t}, w_{t}\right)$.

In Theorem 1 below, part (ii) shows that the problem facing the decision maker in each period is the maximization of a concave function, which is property (a). Part (iii) establishes two properties of the optimal decision in each period - that the optimal withdrawal quantity increases in the groundwater stock, and that the groundwater stock in the next period is nondecreasing in the groundwater stock in the current period - which are Properties (b) and (c), respectively. We prove these results by showing that a modification of $V_{t}$ exhibits the concavity property - which is the content of part (iv).

Theorem 1. Under model (2),

(i) $V_{t}\left(x_{t}\right)$ is nondecreasing in $x_{t}$ for each $t \in\{1, \ldots, T+1\}$,

(ii) $U_{t}\left(x_{t}, w_{t}\right)$ is concave in $w_{t}$ for any $x_{t}$ for each $t \in\{1, \ldots, T\}$,

(iii) $w_{t}^{*}$ satisfies $w_{t}^{*}\left(x_{t}\right) \leq w_{t}^{*}\left(x_{t}+\epsilon\right) \leq w_{t}^{*}\left(x_{t}\right)+\epsilon$ for any $x_{t}$ and $\epsilon>0$ for each $t \in$ $\{1, \ldots, T\},^{2}$ and

(iv) $V_{t}\left(x_{t}\right)+\int_{z=0}^{x_{t}} \gamma(z) d z$ is concave in $x_{t}$ for each $t \in\{1, \ldots, T+1\}$.

Proof. We proceed by backward induction. As a base case, note that $V_{T+1}$ is a zero function and trivially satisfies (i) and (iv). We assume, as an induction hypothesis, that (i) and (iv) hold for $V_{t+1}$, where $t \in\{1, \ldots, T\}$, and prove (i)-(iv) for $t$.

\footnotetext{
${ }^{2}$ This implies that the derivate of $w_{t}^{*}\left(x_{t}\right)$ with respect to $x_{t}$ should belong to $[0,1]$ if it exists. Also, this derivative exists almost everywhere since $w_{t}$ is bounded, nondecreasing and continuous.
} 
The inductive step for (i) is immediate from (3), i.e.,

$$
U_{t}\left(x_{t}, w_{t}\right)=B\left(w_{t}\right)-\left[\Gamma\left(x_{t}\right)-\Gamma\left(x_{t}-w_{t}\right)\right]+\delta E\left[V_{t+1}\left(x_{t}-w_{t}+R_{t}\right)\right]
$$

where $\Gamma(y)=\int_{z=0}^{y} \gamma(z) d z$ is concave, and $V_{t+1}$ is assumed nondecreasing.

To make an inductive step for (ii), we write (3) as

$$
\begin{aligned}
& U_{t}\left(x_{t}, w_{t}\right) \\
& =\left[B\left(w_{t}\right)-\Gamma\left(x_{t}\right)\right] \\
& +(1-\delta) \Gamma\left(x_{t}-w_{t}\right) \\
& -\delta E\left[\Gamma\left(x_{t}-w_{t}+R_{t}\right)-\Gamma\left(x_{t}-w_{t}\right)\right] \\
& +\delta E\left[V_{t+1}\left(x_{t}-w_{t}+R_{t}\right)+\Gamma\left(x_{t}-w_{t}+R_{t}\right)\right] \text {. }
\end{aligned}
$$

We now explain why each of the lines (4)-(7) is individually a concave function of $w_{t}$. This is certainly true of (4), as $B$ is assumed to be concave. As regards (5), we recall the assumption that $\gamma(z)$ is nonincreasing in $z$. It follows that $\Gamma(y)=\int_{z=0}^{y} \gamma(z) d z$ is concave in $z$, and so $\Gamma\left(x_{t}-w_{t}\right)$ is concave. As regards (6), the assumption that $\gamma(z)$ is convex implies that $\partial\left[\Gamma\left(v+R_{t}\right)-\Gamma(v)\right] / \partial v=\gamma\left(v+R_{t}\right)-\gamma(v)$ is nondecreasing in $v$. This implies that $\left[\Gamma\left(v+R_{t}\right)-\Gamma(v)\right]$ is convex in $v$, as is $E\left[\Gamma\left(x_{t}-w_{t}+R_{t}\right)-\Gamma\left(x_{t}-w_{t}\right)\right]$. Finally, it is the inductive hypothesis for (iv) that ensures that (7) is concave in $w_{t}$. We have now established the inductive step for (ii).

We now turn to the inductive step for (iii). This follows from Topkis's theorem [17], which states that if $f(u, v)$ is a supermodular function of $(u, v)$ and $D$ is a lattice then $\arg \max _{v \in D} f(u, v)$ is nondecreasing in $u$. Topkis's theorem applies to our problem since we are seeking to maximize a function of the form $f_{t}\left(x_{t}, w_{t}\right)=U_{t}\left(x_{t}, w_{t}\right)+\Gamma\left(x_{t}\right)=B\left(w_{t}\right)+$ $g_{t}\left(x_{t}-w_{t}\right)$, where both $B$ and $g_{t}$ are concave functions, taking $g_{t}$ to be the sum of lines (5)-(7). A function $h$ is said to be supermodular if $h(a \vee b)+h(a \wedge b) \geq h(a)+h(b)$ (where $a \vee b$ and $a \wedge b$ denote maximum and minimum of $a$ and $b$ ). So, $f_{t}$ is a supermodular function of $\left(x_{t}, w_{t}\right)$ because, for any positive $\epsilon$ and $\delta$,

$$
\begin{aligned}
f_{t}\left(x_{t}+\epsilon, w_{t}+\delta\right)+f\left(x_{t}, w_{t}\right) & \geq f_{t}\left(x_{t}+\epsilon, w_{t}\right)+f\left(x_{t}, w_{t}+\delta\right) \\
& \Longleftrightarrow g_{t}\left(x_{t}+\epsilon-w_{t}-\delta\right)+g_{t}\left(x_{t}-w_{t}\right) \geq g_{t}\left(x_{t}+\epsilon-w_{t}\right)+g_{t}\left(x_{t}-w_{t}-\delta\right)
\end{aligned}
$$

and this is implied by the concavity of $g_{t}$. Thus, Topkis's theorem tells us that $w_{t}^{*}\left(x_{t}+\epsilon\right) \geq$ $w_{t}^{*}\left(x_{t}\right)$. To show $w_{t}^{*}\left(x_{t}+\epsilon\right) \leq w_{t}^{*}\left(x_{t}\right)+\epsilon$, which is equivalent to $\left(x_{t}+\epsilon\right)-w_{t}^{*}\left(x_{t}+\epsilon\right) \geq x_{t}-$ 
$w_{t}^{*}\left(x_{t}\right)$, we need only make the same application of Topkis's theorem, but take the function to be maximized $\varphi_{t}\left(x_{t}, y_{t}\right)=B\left(x_{t}-y_{t}\right)+g_{t}\left(y_{t}\right)$, making the identification of $y_{t}=x_{t}-w_{t}$.

Finally, to make the inductive step for (iv), we consider the equation involving (4)-(7), move $\Gamma\left(x_{t}\right)$ to the left hand side, and replace $w_{t}$ with $w_{t}^{*}\left(x_{t}\right)$. This gives

$$
V_{t}\left(x_{t}\right)+\Gamma\left(x_{t}\right)=B\left(w_{t}^{*}\left(x_{t}\right)\right)+g_{t}\left(x_{t}-w_{t}^{*}\left(x_{t}\right)\right) .
$$

Note that since $w_{t}^{*}\left(x_{t}\right)$ maximizes $U_{t}\left(x_{t}, w_{t}\right)=B\left(w_{t}\right)-\Gamma\left(x_{t}\right)+g_{t}\left(x_{t}-w_{t}\right)$ with respect to $w_{t}$, it is also the value of $w$ maximizing

$$
B(w)+g_{t}\left(x_{t}-w\right)
$$

subject to $w \geq 0$. Since $B$ and $g_{t}$ are concave, the expression in (8) is jointly concave in $x_{t}$ and $w$. Thus,

$$
B\left(w_{t}^{*}\left(x_{t}\right)\right)+g_{t}\left(x_{t}-w_{t}^{*}\left(x_{t}\right)\right)=\max _{w \geq 0} B(w)+g_{t}\left(x_{t}-w\right)
$$

is concave in $x_{t}$ (See, for example, Section 3.2.5 of [4]). Therefore, we conclude that $V_{t}\left(x_{t}\right)+$ $\Gamma\left(x_{t}\right)$ is concave, proving (iv).

We note that an alternative way to prove Property (c) is to use a sample path argument: if $x^{1}<x^{2}$ and $x^{1}-w^{1}>x^{2}-w^{2}$ hold for some period $t$, then replacing $w^{1}$ with $w^{1}+x^{2}-x^{1}$ and $w^{2}$ with $w^{2}-x^{2}+x^{1}$ results in a solution that is not worse than the original solution this occurs because of the supermodularity of $B(x-y)-C(x, x-y)$ in $(x, y)$.

The following result shows that with more periods to go, the optimal decision is more conservative in the extraction of water (Property (d)), and implies in particular that the optimal extraction quantity is bounded above by the myopic withdrawal quantity (which corresponds to the last period).

Corollary 1. Under model (2), $V_{t}(x)$ is submodular in $(t, x)$, i.e., $V_{t^{1}}\left(x^{1}\right)+V_{t^{2}}\left(x^{2}\right) \leq$ $V_{t^{1}}\left(x^{2}\right)+V_{t^{2}}\left(x^{1}\right)$ whenever $t^{1} \geq t^{2}$ and $x^{1} \geq x^{2}$. Furthermore, for any $x$ and $t \leq T$, $w_{t}^{*}(x) \leq w_{t+1}^{*}(x)$.

Proof. We remind the reader

$$
U_{t}\left(x_{t}, w_{t}\right)=B\left(w_{t}\right)-\left[\Gamma\left(x_{t}\right)-\Gamma\left(x_{t}-w_{t}\right)\right]+\delta E\left[V_{t+1}\left(x_{t}-w_{t}+R_{t}\right)\right],
$$


where $\Gamma(y)=\int_{z=0}^{y} \gamma(z) d z$, and

$$
V_{t}\left(x_{t}\right)=\max _{w_{t} \geq 0} U_{t}\left(x_{t}, w_{t}\right)
$$

with $V_{T+1}\left(x_{T+1}\right)=0$. To show the submodularity of $V_{t}(x)$ in $(t, x)$, we need to show, for any $1<t \leq T+1$ and any pair of $x^{1}$ and $x^{2}$ satisfying $x^{2}<x^{1}$,

$$
\Delta_{t}\left(x^{1}, x^{2}\right)=V_{t}\left(x^{1}\right)+V_{t-1}\left(x^{2}\right)-V_{t-1}\left(x^{1}\right)-V_{t}\left(x^{2}\right) \leq 0 .
$$

As a base case, note that $\Delta_{T+1}\left(x^{1}, x^{2}\right) \leq 0$ holds since $V_{T+1}$ is a zero function, and $V_{T}$ is a nondecreasing function (Theorem 1(a)). We proceed with backward induction on $t$, by assuming $\Delta_{t+1}\left(x^{1}, x^{2}\right) \leq 0$ for any $x^{2}<x^{1}$, where $1<t \leq T$. Let $w_{t}^{1}=w_{t}^{*}\left(x^{1}\right)$ and $w_{t-1}^{2}=w_{t-1}^{*}\left(x^{2}\right)$.

CASE $x^{1}-w_{t}^{1} \geq x^{2}-w_{t-1}^{2}$. From the definitions of $w_{t}^{1}$ and $w_{t-1}^{2}$, we obtain $V_{t-1}\left(x^{1}\right)=\max _{w \geq 0} U_{t-1}\left(x^{1}, w\right) \geq U_{t-1}\left(x^{1}, w_{t}^{1}\right) \quad$ and $\quad V_{t}\left(x^{2}\right)=\max _{w \geq 0} U_{t}\left(x^{2}, w\right) \geq U_{t}\left(x^{2}, w_{t-1}^{2}\right)$. Thus,

$$
\begin{aligned}
\Delta_{t}\left(x^{1}, x^{2}\right) \leq & U_{t}\left(x^{1}, w_{t}^{1}\right)+U_{t-1}\left(x^{2}, w_{t-1}^{2}\right)-U_{t-1}\left(x^{1}, w_{t}^{1}\right)-U_{t}\left(x^{2}, w_{t-1}^{2}\right) \\
= & \delta E\left[V_{t+1}\left(x^{1}-w_{t}^{1}+R_{t}\right)+V_{t}\left(x^{2}-w_{t-1}^{2}+R_{t-1}\right)\right. \\
& \left.\quad-V_{t}\left(x^{1}-w_{t}^{1}+R_{t-1}\right)-V_{t+1}\left(x^{2}-w_{t-1}^{2}+R_{t}\right)\right] .
\end{aligned}
$$

Since $R_{t}$ and $R_{t-1}$ have an identical distribution, we apply the induction hypothesis to conclude the rightmost expression above is at most zero. It follows that $\Delta_{t}\left(x^{1}, x^{2}\right) \leq 0$.

CASE $x^{1}-w_{t}^{1}<x^{2}-w_{t-1}^{2}$. Recall $x^{1}>x^{2}$. It follows immediately that both $w_{t-1}^{2}+x^{1}-$ $x^{2}$ and $w_{t}^{1}-x^{1}+x^{2}$ are nonnegative. By using an argument similar to the one used in the previous case,

$$
V_{t-1}\left(x^{1}\right) \geq U_{t-1}\left(x^{1}, w_{t-1}^{2}+x^{1}-x^{2}\right) \quad \text { and } \quad V_{t}\left(x^{2}\right) \geq U_{t}\left(x^{2}, w_{t}^{1}-x^{1}+x^{2}\right)
$$

Then, from the definition of $U_{t}$,

$$
\begin{aligned}
\Delta_{t}\left(x^{1}, x^{2}\right) & \leq U_{t}\left(x^{1}, w_{t}^{1}\right)+U_{t-1}\left(x^{2}, w_{t-1}^{2}\right)-U_{t-1}\left(x^{1}, w_{t-1}^{2}+x^{1}-x^{2}\right)-U_{t}\left(x^{2}, w_{t}^{1}-x^{1}+x^{2}\right) \\
& =B\left(w_{t}^{1}\right)+B\left(w_{t-1}^{2}\right)-B\left(w_{t-1}^{2}+x^{1}-x^{2}\right)-B\left(w_{t}^{1}-x^{1}+x^{2}\right)
\end{aligned}
$$

where the right-hand most expression is at most zero by the concavity of $B$ and $w_{t-1}^{2} \leq$ $\min \left\{w_{t-1}^{2}+x^{1}-x^{2}, w_{t}^{1}-x^{1}+x^{2}\right\} \leq \max \left\{w_{t-1}^{2}+x^{1}-x^{2}, w_{t}^{1}-x^{1}+x^{2}\right\} \leq w_{t}^{1}$. 
Thus, we complete the induction step, implying the submodularity of $V_{t}$ for each $t$. Now, since $V_{t}(x)$ is submodular in $(t, x)$, by applying the Topkis's theorem used in the proof of Theorem 1, we obtain that $w_{t}^{*}(x)$ is nondecreasing in $t$. To provide the details of this argument, suppose by way of contradiction that $w_{t}^{*}(x)>w_{t+1}^{*}(x)$ for some $t$ and $x$. Let $w_{0}=w_{t}^{*}(x)$ and $w_{1}=w_{t+1}^{*}(x)$. Then, the sums of the expected payoffs satisfy

$$
\begin{aligned}
& U_{t}\left(x, w_{0}\right)+U_{t+1}\left(x, w_{1}\right) \\
& =B\left(w_{0}\right)-\left[\Gamma(x)-\Gamma\left(x-w_{0}\right)\right]+\delta E\left[V_{t+1}\left(x-w_{0}+R_{t}\right)\right] \\
& +B\left(w_{1}\right)-\left[\Gamma(x)-\Gamma\left(x-w_{1}\right)\right]+\delta E\left[V_{t+2}\left(x-w_{1}+R_{t+1}\right)\right] \\
& \leq B\left(w_{0}\right)-\left[\Gamma(x)-\Gamma\left(x-w_{1}\right)\right]+\delta E\left[V_{t+1}\left(x-w_{1}+R_{t}\right)\right] \\
& +B\left(w_{1}\right)-\left[\Gamma(x)-\Gamma\left(x-w_{0}\right)\right]+\delta E\left[V_{t+2}\left(x-w_{0}+R_{t+1}\right)\right] \\
& =U_{t}\left(x, w_{1}\right)+U_{t+1}\left(x, w_{0}\right) \text {, }
\end{aligned}
$$

where the inequality follows from the submodularity property of $V_{t}(x)$. However, this contradicts the choice of $w_{t}^{*}(x)$ and $w_{t+1}^{*}(x)$.

REMARK: In the paper, we have assumed that $C(x, w)=\int_{z=x-w}^{x} \gamma(z) d z=\Gamma(x)-\Gamma(x-w)$. It is evident (from an inspection of the proof of Theorem 1) that, for our analysis, the differentiability of $\Gamma$ is not required. Indeed, it is sufficient if $C(x, w)=\Gamma(x)-\Gamma(x-w)$, with $\Gamma$ nondecreasing and concave and $\Gamma(v+R)-\Gamma(v)$ convex in $v$ for any $R>0$.

We also note that the proof of Theorem 1 can be generalized in a straightforward manner to the case where the $R_{t}$ 's are no longer independent or identically distributed (i.i.d.). However, this i.i.d. property is required for the proof of Corollary 1.

\section{Other Problems of Sequence Resource Allocation}

It is interesting to observe that the results in this paper are very similar to those that have been obtained for some other well-studied problems of operations research. One of these is the "fighter problem", discussed in $[2,3,20]$. In this problem a fighter airplane is initially endowed with a quantity of missiles and wishes to ration its use of these so as to maximize the expected number of randomly encountered enemy planes that it can shoot down during a given time T. A special case is that of the "invincible fighter", which is not itself liable to being shot down by the enemy planes. The discrete-time continuous-ammunition version of 
the invincible fighter problem corresponds to a special case of our model (with $C(x, w)=0$, $R_{t}=0, \delta=1 ; B(w)$ is the probability of shooting down an enemy plane by expending a quantity of ammunition $w$ ). It has been shown in [2] and [3] that properties called (A), (B) and $(\mathrm{C}$ ) hold for the invincible fighter; these are analogous, respectively, to the Properties (d), (b) and (c) that we have established in Section 1.

\section{Acknowledgement}

We thank Sha Liao and Juan Serpa for correcting typographical errors.

\section{References}

[1] R. M. Aggarwal and T. A. Narayan. Does inequality lead to greater efficiency in the use of local commons? The role of strategic investments in capacity. Journal of Environmental Economics and Management, 47(1):163-182, 2004.

[2] J. Bartroff, L. Goldstein, Y. Rinott, and E. Samuel-Cahn. On optimal allocation of a continuous resource using an iterative approach and total positivity. Advances in Applied Probability, 42(3):795-815, 2010.

[3] J. Bartroff and E. Samuel-Cahn. The fighter problem: optimal allocation of a discrete commodity. Advances in Applied Probability, 43(1):1-10, 2011.

[4] S. Boyd and L. Vandenberghe. Convex Optimization. Cambridge University Press, Cambridge, United Kingdom, 2004.

[5] G. Brown, Jr. and R. Deacon. Economic optimization of a single-cell aquifer. Water Resources Research, 8(3):557-564, 1972.

[6] H.S. Burness and T.C. Brill. The role for policy in common pool groundwater use. Resource and energy economics, 23(1):19-40, 2001.

[7] O. R. Burt. Optimal resource use over time with an application to ground water. Management Science, 11(1):80-93, 1964.

[8] O. R. Burt. Economic control of groundwater reserves. Journal of Farm Economics, 48(3):632-647, 1966. 
[9] O. R. Burt. Temporal allocation of groundwater. Water Resources Research, 3(1):45-56, 1967.

[10] O. R. Burt. Groundwater storage control under institutional restriction. Water Resources Research, 6(6):45-56, 1970.

[11] M. Gisser and D. A. Sanchez. Competition versus optimal control in groundwater pumping. Water Resources Research, 16(4):638-642, 1980.

[12] P. Hellegers, D. Ziberman, and E. van Ierland. Dynamics of agricultural groundwater extraction. Ecological Economics, 37(2):303-311, 2001.

[13] K. C. Knapp and L. J. Olson. The economics of conjunctive groundwater management with stochastic surface supplies. Journal of Environmental Economics and Management, 28(3):340-356, 1995.

[14] B. Provencher and O. Burt. The externalities associated with the common property exploitation of groundwater. Journal of Environmental Economics and Management, 24(2):139-158, 1993.

[15] C. Roseta-Palma and A. Xepapadeas. Robust control in water management. Journal of Risk and Uncertainty, 29(1):21-34, 2004.

[16] S. J. Rubio and J. P. Castro. Long run groundwater reserves under uncertainty. investigaciones económicas, 20(1):71-88, 1996.

[17] D. M. Topkis. Minimizing a submodular function on a lattice. Operations Research, 26(2):305-321, 1978.

[18] Y. Tsur and T. Graham-Tomasi. The buffer value of groundwater with stochastic water supplies. Journal of Environmental Economics and Management, 21(3):201-224, 1991.

[19] Y. Tsur and A. Zemel. Endangered aquifers: Groundwater management under threats of catastrophic events. Water Resource Research, 40, 2004. 06S20, doi:10.1029/2003WR002168.

[20] R. R. Weber. A problem of ammunition rationing. In F. J. Radermacher, G. Ritter, and S. M. Ross, editors, Conference report: Stochastic Dynamic Optimization and Applications in Scheduling and Related Field, page 148, held at University of Passau, Facultät für Mathematik und Informatik, 1985. 
[21] V. E. Worthington, O. R. Burt, and R. L. Brustkern. Optimal management of a confined groundwater system. Journal of Environmental Economics and Management, 12(3):229$245,1985$.

[22] N. Zeitouni. Optimal extraction from a renewable groundwater aquifer with stochastic recharge. Water Resources Research, 40(6):W06S19, 2004. 